Vol. $14 \mathrm{~N}^{\circ} 1$, pp. 43-50, July 1995

Universidad Católica del Norte

Antofagasta - Chile

\title{
A CHARACTERIZATION OF LORENTZ-IMPROVING MEASURES
}

\author{
RAYMOND J. GRINNELL1 \\ University of the West Indies Bridgetown, Barbados, West Indies
}

\begin{abstract}
Let $G$ be an infinite compact abelian group and let $\Gamma$ denote its dual group. A borel measure $\mu$ on $G$ is called Lorentz-improving if there existe $p, q_{1}$, and $q_{2}$, where $1<p<\infty$ and $1 \leq q_{1} \leq q_{2} \leq \infty$, such that $\mu * L\left(p, q_{2}\right) \subseteq L\left(p, q_{1}\right)$. A detailed exposition of our recent characterization of Lorentz-improving measures is presented here. In this result Lorentz-improving measures are characterized in terms of the size of the sets $\{\gamma \in \Gamma:|\hat{\mu}(\gamma)|>\epsilon\}$ and in terms of $n$-fold convolution powers. This characterization is analogous to a known characterization of $L^{p}$-improving measures due to Hare.
\end{abstract}

1991 Mathematics Subject Classification: Primary 43A05; Secondary $43 A 25,43 A 15$.

\footnotetext{
${ }^{1}$ Research supported by UWI Study and Travel Grant.
} 


\section{Introduction.}

The purpose of this article is to present in detail a proof of our recent characterization of Lorentz-improving measures [2, Theorem 3.4]. Although a statement of this result is given in that paper, one finds only an outline and references to the provif. Thus an exposition of the particulars of the argument is in order.

Throughout this paper $G$ denotes an infinite compact abelian group, $\lambda$ its normalized Haar measure, and $\Gamma$ its dual group. Let $M(G)$ denote the complex bounded regular Borel measures on $G$ and let $T(G)$ denote the trigonometric polynomials on $G$. For $1 \leq p \leq \infty, L^{p}$ denotes the usual Lebesgue space $L^{p}(G)$ and for $1 \leq q \leq \infty, L(p, q)$ denotes the Lorentz space $L(p, q)(G)$. Denote the norm on $L(p, q)$ by $\|\cdot\|_{(p, q)}$ and the quasinorm by $\|\cdot\|_{p, q}^{*}$. Lorentz spaces and Lebesgue spaces are related by the proper inclusions

$$
\begin{aligned}
& L^{\infty} \subset \cup_{t>p} L^{t} \subset L(p, q) \subset L(p, p)= \\
& \quad=L^{p} \subset L(p, r) \subset \cap_{s<p} L^{s} \subset L^{1}
\end{aligned}
$$

whenever $1<q<p<r<\infty$. As well, the norm and quasi-norm are related by

$$
\left(\frac{p}{q}\right)^{\frac{1}{q}}\|f\|_{p, q}^{*} \leq\|f\|_{(p, q)} \leq p^{\prime}\left(\frac{p}{q}\right)^{\frac{1}{q}}\|f\|_{p, q}^{*}
$$

whenever $1<p<\infty$ and $1 \leq q \leq \infty$ and $p^{\prime}$ is the index conjugate to $p$ (if $q=\infty$ then $\left(\frac{p}{q}\right)^{\frac{1}{q}}=1$ ). Further details on Lorentz spaces can be found in [2].

Here is the main definition. A measure $\mu \in M(G)$ is called Lorentzimproving if there exists $p, q_{1}$, and $q_{2}$, where $1<p<\infty$ and $1 \leq q_{1}<q_{2} \leq$ $\infty$, such that $\mu * L\left(p, q_{2}\right) \subseteq L\left(p, q_{1}\right)$. Equivalently, $\mu$ is Lorentz-improving if there exists a constant $k$ such that

$$
\|\mu * f\|_{\left(p, q_{1}\right)} \leq k\|f\|_{\left(p, q_{2}\right)} \text { for all } f \in L\left(p, q_{2}\right)
$$

An original motivation for studying these measures can be traced to the theory or $L^{p}$-improving measures which have been investigated in a number of papers (see [1], [4], and the references cited therein). Lorentz-improving measures are first discussed in [3] and further in [2]. The main result of this 
present paper, namely the proof of Theorem 3.4 of [2], is a generalization of a known characterization of $L^{p}$-improving measures found in [4].

\section{The Characterization Theorem.}

If $X \subseteq L^{1}$ and $E \subseteq \Gamma$ let $X_{E}=\{f \in X: \hat{f}(\gamma)=0$ for all $\gamma \notin E\}$. Recall that $E$ is a $\Lambda(p)$ set for some $1<p<\infty$ if $L_{E}^{p}=L_{E}^{1}$. The main result in [4] states that $\mu$ is $L^{p}$-improving if and only if the sets $\{\gamma \in \Gamma:|\dot{\mu}(\gamma)>\epsilon|\}$ are $\Lambda(p)$ sets for some $p>2$, with $\Lambda(p)$ constant $O\left(\epsilon^{-1}\right)$. The following is a generalization of $\Lambda(p)$ sets and is used in the main result.

Let $1<p<\infty$ and $1 \leq q<\infty$. A set $E \subseteq \Gamma$ is called a $\Lambda_{1}(p, q)$ set if there exists some $1<s<p$ such that $L_{E}(p, q)=L_{E}(s, q)$ and is called a $\Lambda_{2}(p, q)$ set if there exists some $q<r<\infty$ such that $L_{E}(p, q)=L_{E}(p, r)$.

If $E$ is a finite set then for each $1<p<\infty$ and $1 \leq q<\infty, L_{E}(p, q)=$ $T_{E}(G)$ and so $E$ is both a $\Lambda_{1}(p, q)$ set and a $\Lambda_{2}(p, q)$ set. The group $\Gamma$ itself is not a $\Lambda 1(p, q)$ set or a $\Lambda_{2}(p, q)$ set for any such $p$ and $q$ since $L(p, q) \neq L(s, q)$ if $1<s<p$ and $L(p, q) \neq L(p, r)$ if $q<r<\infty$ (see $\left[2\right.$, p.368]). We use specifically $\Lambda_{2}(p, q)$ sets in the main result below. If $\mu \in M(G)$ and $\epsilon>0$ let $E(\mu, \epsilon)=\{\gamma \in \Gamma:|\hat{\mu}(\gamma)>\epsilon|\}$ and let $\mu^{n}$ denote the $n$-fold convolution of $\mu$ with itself. If $E \subseteq \Gamma$ and $1 \leq q<2$ let $\Lambda_{2}(2, q ; E)=\sup \left\{\|f\|_{(2, q)}: f \in L_{E}^{2},\|f\|_{2} \leq 1\right\}$. Here is the main result.

Theorem [2, Theorem 3.4]. For a measure $\mu \in M(G)$ the following assertions are equivalent:

(i) $\mu$ is Lorentz-improving;

(ii) There exists $1 \leq q<2$ such that for each $\epsilon>0$ the sets $E(\mu, \epsilon)$ are $\Lambda_{2}(2, q)$ sets and $\Lambda_{2}(2, q ; E(\mu, \epsilon)) \leq O\left(\epsilon^{-1}\right) ;$

(iii) There exists $1 \leq q<2$ and an $\alpha \geq 1$ such that for each $\epsilon>0$ the sets $E(\mu, \epsilon)$ are $\Lambda_{2}(2, q)$ sets and $\Lambda_{2}(2, q ; E(\mu, \epsilon)) \leq O\left(\epsilon^{-\alpha}\right)$;

(iv) There exists an integer $n \geq 2$ such that $\mu^{n}$ is Lorentz-improving.

The proof of this theorem requires three lemmas.

Lemma 1. If $\mu \in M(G)$ and $n$ is a positive integer then for each $z \in \mathbf{C}$ there exists an operator $T_{z}$ on $L^{2}$ such that $T_{z}(f)^{\wedge}(\gamma)=\hat{f}(\gamma)|\hat{\mu}(\gamma)|^{n z}$ for each $\gamma \in \Gamma$. 
Proof. Let $\operatorname{supp}(\hat{\mu})=\{\gamma \in \Gamma: \dot{\mu}(\gamma) \neq 0\}$ and let $f \in L^{2}$. A calculation using Plancherel's theorem shows that the sum $\left.\left.\sum_{\gamma \in \operatorname{supp}(\dot{\mu})}|\hat{f}(\gamma)| \dot{\mu}(\gamma)\right|^{n z}\right|^{2}$ is finite for each $z \in \mathbf{C}$. Another application of Plancherel's theorem shows there is an unique operator $T_{z}$ on $L^{2}$ defined via its Fourier transform which satisfies the asserted condition.

Let $P=\{z \in \mathbf{C}: \operatorname{Re}(z) \geq 0\}, \Omega=\{z \in \mathbf{C}: 0<\operatorname{Re}(z)<1\}$, and $\bar{\Omega}=$ $\{z \in \mathbf{C}: 0 \leq \operatorname{Re}(z) \leq 1\}$.

Lemma 2. Let $\mu \in M(G)$, let $n$ be a positive integer, and let $f, g \in L^{2}$. Then the function $F$ defined on $P$ by $F(z)=\int_{G} T_{z}(f)(s) g(s) d \lambda(s)$ is continous on $\bar{\Omega}$ and analytic on $\Omega$.

Proof. Let $\left\{\gamma_{j}\right\}$ be an enumeration of the set $\operatorname{supp}(\hat{\mu}) \cap \operatorname{supp}(\hat{f})$. It follows from lemma 1 and Parseval's identity that

$$
F(z)=\sum_{j=1}^{\infty} \hat{f}\left(\gamma_{j}\right)\left|\hat{\mu}\left(\gamma_{j}\right)\right|^{n z} \overline{\hat{g}\left(\gamma_{j}\right)}
$$

for each $z \in P$. If for each positive integer $k, F_{k}$ denotes the kth partial sum of $F$, then $\left\{F_{k}\right\}$ converges uniformly to $F$ on compact subsets of $\bar{\Omega}$ and $\Omega$.

This is sufficient to give the result.

Lemma 3. If $E \subseteq \Gamma$ anf if $1<p<\infty$ and $1 \leq q<\infty$ then $T_{E}(G)$ is a dense subspace of $L_{E}(p, q)$.

Proof. It is clear that we need only verify the density property. Let $f \in L_{E}(p, q)$ and let $\left\{h_{\alpha}\right\}$ be a bounded approximate identity for $L^{1}$ consisting of elements in $T(G)$. If $\gamma \notin E$ then $\left(h_{\alpha} * f\right)^{\wedge}(\gamma)=h_{\alpha}(\gamma) f(\gamma)=0$ so $h_{\alpha} * f \in T_{E}(G)$. It follows from [5, Remarks 32.33 (a), (d)] that $\lim _{\alpha}\left\|h_{\alpha} * f-f\right\|_{(p, q)}=0$ which gives the result. $\square$

\section{Proof of the Theorem.}

(i $\Rightarrow$ ii) The assumption of (i) and [2, Theorem 1.4 (a)] implies there is a constant $k$ and $1 \leq q<2$ such that $\|\mu * f\|_{(2, q)} \leq k\|f\|_{2}$ for all $f \in L^{2}$. For $\epsilon>0$ and $f \in T_{E(\mu, \epsilon)}(G)$ define a function $g$ by 


$$
\hat{g}(\gamma)=\left\{\begin{array}{cc}
\frac{\hat{f}(\gamma)}{\hat{\mu}(\gamma)} & \text { if } \gamma \in E(\mu, \epsilon) \\
0 & \text { otherwise. }
\end{array}\right.
$$

Clearly $g \in T_{E(\mu, \epsilon)}(G)$ and $\mu * g=f$. A straightforward calculation shows that $\|g\|_{2} \leq \frac{1}{\epsilon}\|f\|_{2}$. By combining these inequalities and using (1.2) we obtain that $\|f\|_{(2, q)} \leq \frac{2 k}{\epsilon}\|f\|_{(2,2)}$ for $f \in T_{E(\mu, \epsilon)}(G)$. Assertion (ii) now follows from Lemma 3 and the definition of a $\Lambda_{2}(p, q)$ set.

(ii $\Rightarrow$ iii) This is obvious with $\alpha=1$.

(iii $\Rightarrow$ iv) There is no loss of generality in assuming that $\|\mu\| \leq 1$ and so $E(\mu, \epsilon)$ is empty if $\epsilon>1$. For each positive integer $j$, let $E_{j}=$ $\left\{\gamma \in \Gamma: 2^{-j}<|\hat{\mu}(\gamma)| \leq 2^{1-j}\right\}$. Since $E_{j} \subseteq E\left(\mu, 2^{-j}\right)$, (iii) implies that $E_{j}$ is a $\Lambda_{2}(2, q)$ set for some $1 \leq q<2$ and $\alpha \geq 1$ and $\Lambda_{2}\left(2, q ; E_{j}\right) \leq 2^{\alpha j} k$ for some constant $k$. A calculation using [2, Theorem 3.3] and (1.2) shows that for each positive integer $j$ and $f \in L\left(2, q^{\prime}\right)$,

$$
\sum_{\gamma \in E_{j}}|\hat{f}(\gamma)|^{2} \leq 64\left(2^{\alpha j} k\right)^{2}\left(\|f\|_{2, q^{\prime}}^{*}\right)^{2}
$$

where $q^{\prime}$ is the index conjugate to $q$.

Note that if $\gamma \in E_{j}$ then $|\dot{\mu}(\gamma)| \leq 2^{1-j}$ and so for each positive integer $n,\left|(\hat{\mu}(\gamma))^{n}\right|_{\infty} \leq 2^{n(1-j)}$. As well, since we need only consider $0<\epsilon \leq 1$, $\operatorname{supp}(\hat{\mu})=\bigcup_{j=1}^{\infty} E_{j}$. If $f \in L^{2}$ then the inequalities above and Plancherel's theorem show that

$$
\begin{gathered}
\left\|\mu^{n} * f\right\|_{2}^{2}=\sum_{j=1}^{\infty}\left(\sum_{\gamma \in E_{j}}\left|(\hat{\mu}(\gamma))^{n}\right|^{2}|\hat{f}(\gamma)|^{2}\right) \leq \sum_{j=1}^{\infty} 2^{2 n(1-j)}\left(\sum_{\gamma \in E}|\hat{f}(\gamma)|^{2}\right) \\
\leq \sum_{j=1}^{\infty} 2^{2 n(1-j)} 64\left(2^{\alpha j} k^{2}\right)\left(\|f\|_{2, q^{\prime}}^{*}\right)^{2}
\end{gathered}
$$


If $n>\alpha$ and $C=\sum_{j=1}^{\infty} 2^{2 n(1-j)} 2^{\alpha j}$ then $C$ is finite and $\left\|\mu^{n} * f\right\|_{2} \leq$. $8 C^{\frac{1}{2}} k\|f\|_{2, q^{\prime}}^{*}$. This proves (iv).

(iv $\Rightarrow$ i) Assertion (iv) implies there is an integer $n \geq 2$, a constant $k$, and $1 \leq q<2$ such that $\left\|\mu^{n} * f\right\|_{2} \leq k\|f\|_{2, q}^{*}$ for all $f \in L(2, q)$. The main tool in this part of the proof is an interpolation theorem for operators on Lorentz spaces due to Sagher [7, Main Theorem]. For each $z \in \bar{\Omega}$ let $T_{z}$ be the linear map as defines in Lemma 1 where $n$ is as above and let $\mathcal{F}=\left\{T_{z}: z \in \bar{\Omega}\right\}$. In order to use the interpolation theorem we must first verify the technical conditions that $\mathcal{F}$ is an analytic family of operators and of admissible growth (see [7, p.350]). For a $\lambda$-measurable set $A \subseteq G$ and a $t \in G$ let $\emptyset_{A, t}$ be the function defined on $\bar{\Omega}$ by $\emptyset_{A, t}(z)=T_{z}\left(\chi_{A}\right)(t)$ where $\chi_{A}$ denotes the characteristic function of $A$. It follows from Lemma 2 that for each $g \in L^{2}$ the function $F(z)=\int_{G} \emptyset_{A, t}(z) g(t) d \lambda(t)$ is continous on $\bar{\Omega}$ and analytic on $\dot{\Omega}$. Now $[6$, Appendix, 4$]$ shows that $\emptyset_{A, t}$ is continous on $\bar{\Omega}$ and analytic on $\Omega$ which means $\mathcal{F}$ is an analytic family. To show that $\mathcal{F}$ is of admissible growth, we must verify that for each $y \in \mathbf{R}$,

$$
\sup _{x \in[0,1]} \log \left|\emptyset_{A, t}(x+i y)\right| \leq B e^{b|y|}
$$

where $b, B$ are constants and $b<\pi$. Let $\mathcal{B}\left(L^{\infty}\right)$ denote the bounded linear operators on $L^{\infty}$ and let $\|\cdot\|_{\text {op }}$ denote the norm in $\mathcal{B}\left(L^{\infty}\right)$. Observe that $T_{0}=I$ is the identity in $\mathcal{B}\left(L^{\infty}\right)$ and if $\delta>0$ is sufficiently small, then setting $T=T_{\delta}$ it follows that $T \in \mathcal{B}\left(L^{\infty}\right)$ and $\|I-T\|_{o p}<1$. This implies that $\log (T)=\sum_{n=1}^{\infty} \frac{(-1)^{n}(I-T)^{n}}{n}$ and $\log (T) \in \mathcal{B}\left(L^{\infty}\right)$ also. For $z \in \bar{\Omega}$, $T_{z}=(T)^{\frac{z}{\delta}}=\exp \left(\frac{z}{\gamma} \log (T)\right)$ and so

$$
\left|\emptyset_{A, t}(x+i y)\right|=\left|\emptyset_{A, t}(z)\right| \leq\left\|T_{z}\left(\chi_{A}\right)\right\|_{\infty}=\left\|\exp \left(\frac{|z|}{\delta} \log (T)\right)\left(\chi_{A}\right)\right\|_{\infty}
$$

Now $\left\|\log (T)\left(\chi_{A}\right)\right\|_{\infty} \leq\|\log (T)\|_{o p}$ and

$$
\left\|\exp \left(\frac{z}{\gamma} \log (T)\right)\left(\chi_{A}\right)\right\|_{\infty} \leq \exp \left(\frac{|z|}{\gamma}\|\log (T)\|_{o p}\right)
$$

and thus $\log \left|\varnothing_{A, t}(x+i y)\right| \leq \frac{|z|}{\delta}\|\log (T)\|_{\text {op }}$.

It is easy to verify that for $z \in \bar{\Omega},|z| \leq 2 e^{2|y|}$ and so 


$$
\log \left|\emptyset_{A, t}(z)\right| \leq \frac{2}{\delta}|| \log (T) \|_{o p} e^{2|y|}
$$

This clearly proves $\mathcal{F}$ is of admissible growth. Sagher's interpolation theorem now shows that for each $0<\theta<1$,

$$
\left\|T_{0}(f)\right\|_{2} \leq B(\theta)\|f\|_{2, s(\theta)}^{*}
$$

for all $f \in L(2, s(\theta))$ where $S(\theta)$ is defined by the equation $\frac{1}{s(\theta)}=\frac{1-\theta}{2}+$ $\frac{\theta}{q}$ and $B(\theta)$ is a constant wich depends on $\theta$. When $\theta=\frac{1}{n}, T_{\frac{1}{n}}(f)=\mu * f$ and so

$$
\|\mu * f\|_{2} \leq B\left(\frac{1}{n}\right)\|f\|_{2, s\left(\frac{1}{n}\right)}^{*}
$$

for all $f \in L\left(2, s\left(\frac{1}{n}\right)\right)$. Since $s\left(\frac{1}{n}\right)>2, L^{2} \subset L\left(2, s\left(\frac{1}{n}\right)\right)$ by (1.1) and hence $\mu$ is Lorentz-improving.

\section{References}

[1] C. Graham, K. Hare, D. Ritter, The size of $L^{p}$-improving measures, J. Funct. Anal. 84 , 472-495 (1980).

[2] R. Grinnell, K. Hare, Lorentz-improving measures, Ill. J. Math. 38 No. 3, 366-389 (1994).

[3] R. Grinnell, Lorentz-improving mesures on compact abelian groups, Ph.D. dissertation, Queen's University (1991).

[4] K. Hare, A characterization of $L^{p}$-improving measures, Proc. Amer. ;Math. Soc. 102 , 295-299 (1988).

[5] E. Hewitt, K. Ross, Abstract harmonic analysis, Vol. II, SpringerVerlag, New York (1970).

[6] Y. Katznelson, An introduction to harmonic analysis, Dover, New York (1976). 
[7] Y. Sagher, On araiytic familes of operators, Israel, J. Math. 7, 350-356 $(1969)$.

Received: January, 1995.

Raymond J. Grinnell

Department of Mathematic: and Computer Science

University of the West Indie"

Cave Hill Campus

P.O. Box 64

Bridgetown, Bari,ados, Wesi Indies. 